\section{Rohstoffabbau, sonst nichts}

\author{
Die Bundesregierung will bis 2020 den deutschen Kohlenstoff- \\ dioxid-Ausstoß um 40 Prozent senken und die Rohstoff- \\ produktivität im Vergleich zu 1994 verdoppeln. Diese Vorgaben \\ sind schön, nur glaubwürdig sind sie nicht. Zu viele Gesetzes- \\ texte werden den Herausforderungen des 21. Jahrhunderts nicht \\ gerecht. Ein Beispiel: das Bundesberggesetz. \\ Von Daniel Hiß
}

V on wegen rohstoffarmes Land! Rund drei Viertel der in Deutschland benötigten mineralischen Rohstoffe kommen aus heimischen Lagerstätten. Bei Braunkohle, Stein- und Kalisalz, Kalkstein, Gips und Schwefel ist Deutschland Selbstversorger. Der niedersächsische Finanzminister freut sich jährlich über Einnahmen in Höhe von rund 590 Millionen Euro aus der Erdgasindustrie. Fakt ist also: Der Gewinnung von Bodenschätzen kommt in Deutschland eine große wirtschaftliche Bedeutung zu. Jährlich trägt sie rund 20 Milliarden Euro zum Bruttoinlandsprodukt (BIP) bei.

Den Rechtsrahmen für Aufsuchung und Abbau von Rohstoffen setzt in Deutschland das Bundesberggesetz (BBergG), das der Rohstoffgewinnung einen besonderen Vorrang vor allen anderen Interessen einräumt. Insbesondere Umwelt-, Natur- und Ressourcenschutz sowie individuelle Grundrechte finden im B BergG nur wenig Beachtung.

\section{Überholtes Gesetz}

Der Bergbau genießt im deutschen Verwaltungsrecht eine anachronistische Sonderstellung. Angesichts begrenzter Rohstoffvorkommen und ehrgeiziger Ziele in der Klima- und Energiepolitik ist das nicht mehr vertretbar. Eine effektive und schonende Nutzung begrenzter Ressourcen ist elementarer Bestandteil und wichtiges Anliegen der gerade in der Politiksprache oft bemühten Nachhaltigkeit. Ein modernes Bergrecht für das 21. Jahrhundert muss daher Ressourcen- schutz stärker integrieren und den Vorrang des Rohstoffabbaus vor allen anderen Interessen beenden.

\section{Wirtschaftsrecht mit Umweltwirkung}

Der Paragraf 1 des BBergG macht deutlich, was Sinn und Zweck des Gesetzes ist: Es soll die Sicherung der Rohstoffversorgung fördern, Arbeitssicherheit gewährleisten und den Umgang mit Bergbauschäden regeln. Der schonende Umgang mit Umwelt und natürlichen Ressourcen wiederum ist kein vorrangiges Ziel des Bergrechts. Die sogenannte Rohstoffsicherungsklausel des Paragrafen 48 B BergG manifestiert diesen Gesetzeszweck vollends: „Bei Anwendung dieser Vorschriften [Verbotsvorschriften außerhalb des BBergG, Anm. des Autors] ist dafür Sorge zu tragen, dass die Aufsuchung und Gewinnung so wenig wie möglich beeinträchtigt werden." Als Teil des Wirtschaftsverwaltungsrechts dient das BBergG also vor allem dazu, den Ressourcenhunger in Deutschland zu stillen. Ohne erhebliche Folgen für die Umwelt geht das nicht.

Der Abbau von Bodenressourcen ist nicht nur ein Eingriff in das Boden- und Gesteinsgefüge, sondern führt auch zur Zerstörung gewachsener Kulturlandschaft und zum teils dauerhaften Verlust natürlicher Lebensräume für Tiere und Pflanzen. Aber auch für die Menschen ist der Bergbau mit drastischen Konsequenzen verbunden. Allein den großen Braunkohletagbauen mussten seit 1945 in Deutschland über 300 Ortschaften und Hunderttausende Menschen weichen.

Da wirkt es unglaubwürdig, wenn Industrieverbände, Gewerkschaften und Teile der Politik den Schutz von Umwelt und Betroffenen im BBergG als ausreichend bezeichnen. Umweltverträglichkeitsprüfung (UVP) und Ausgleichsmaßnahmen sind im bergrechtlichen Rahmen keine wirksamen Umweltschutzinstrumente, zumal den zuständigen Bergämtern bei der Zulassungsentscheidung kein fachplanerischer Ermessensspielraum bleibt. Das Bundesberggesetz ist alles andere als ein modernes Fachplanungsrecht, das auf einer Gesamtabwägung aller Interessen und einem transparenten Bewilligungsverfahren beruht.

\section{Frackinggesetz - eine verpasste Chance}

Den Mehrheitsfraktionen im Bundestag mangelt es allerdings an politischem Willen, sich an diese wirtschaftspolitisch heilige Kuh zu wagen und eine umfassende Bergrechtsreform anzustoßen. Sicher, im Koalitionsvertrag haben sich CDU, CSU und SPD auf eine Überarbeitung des BBergG in Bezug auf das Wasserhaushaltsgesetz (WHG) verständigt. Die Anfang April auf den Weg gebrachten Frackinggesetze sprechen allerdings eine andere Sprache. Die Regierung will das Bergrecht in dieser Legislaturperiode nicht angehen. Statt den Einsatz von Hydraulic Fracturing zur Gewinnung von Erdöl und Erdgas im B BergG zu regulieren, das die verwaltungsrechtliche Anspruchsgrundlage für die Bewilligung von Abbauvorhaben darstellt, nimmt die Bundesregierung den Umweg über das WHG. Damit schafft sie zwar Genehmigungshürden, mehr als eine ungenügende Behelfsregelung ist das aber nicht.

Das Gesetzespaket zum Fracking ist eine verpasste Chance, das antiquierte Bergrecht an neue technische Herausforderungen anzupassen und für eine grundsätzliche Abwägung zwischen Rohstoffförderung und ökologisch-so- 
zialen Belangen zu öffnen. Immerhin sollen Fracking-Vorhaben künftig UVP-pflichtig sein und eine Ausweitung der Bergschadensvermutung auf den Bohrlochbergbau ist geplant. Das erleichtert im Falle eines Bergschadens die Beweislast zugunsten der Bergbaubetroffenen. Für eine wirksame Schadensregelung fehlt es aber immer noch an verpflichtenden Sicherheitsleistungen und Rückstellungen der Unternehmen, die im Falle einer Insolvenz oder Unternehmensauflösung dem Gläubigerzugriff entzogen sind. Nur so können Schadensregulierung und Rekultivierung nach dem Verursacherprinzip langfristig gewährleistet sein.

\section{Anders wirtschaften}

Neben den Aspekten der Risikominimierung und Umweltvorsorge, wirft die Fracking-Debatte aber auch andere Fragen auf, denen sich die Erdgasunternehmen in Deutschland nur unzureichend stellen. Ist die Gewinnung von unkonventionellen Erdgasreserven überhaupt im Interesse der deutschen Wirtschaft?

Aus einer volkswirtschaftlichen Betrachtungsweise ist das, auch unabhängig vom eingeschlagenen Pfad der Energiewende, klar mit Nein zu beantworten. Analysten der Kreditanstalt für Wiederaufbau kamen 2013 zu dem Ergebnis, dass sich durch den Verzicht auf Fracking in Deutschland kein Standortnachteil ergebe. Das liegt unter anderem an dem relativ geringen Anteil der Energiekosten von rund zwei Prozent an den Gesamtkosten eines Unternehmens. Zudem werden eventuell entstehende Kostennachteile durch höhere Energieeffizienz in der deutschen Wirtschaft ausgeglichen. Angesichts nicht abschätzbarer Folgekosten und eines wachsenden internationalen Gasmarkts mit verschiedenen Anbietern, spricht auch darüber hinaus vieles dafür, die unkonventionellen Erdgasvorräte in Deutschland im Boden zu lassen.

Derartige volkswirtschaftliche Überlegungen sollten bei der Schaffung politischer Rahmenbedingungen eine elementare Rolle spielen. Falsch wäre es, sich allein auf die Wirtschaftlichkeitsüberlegungen der erdgasfördernden Unternehmen zu verlassen. Das skizziert auch die wirtschaftspolitischen Koordinaten für eine Reform des BBergG: Umfassende volkswirtschaftliche Bedarfsprüfungen und das Gebot der Ressourcenschonung sollten Grundlage des Rohstoffabbaus in Deutschland sein, nicht das Profitinteresse einzelner Betriebe.

\section{Anreize zur nachhaltigen Nutzung}

Statt hemmungslosen Raubbau an natürlichen Ressourcen zu befeuern, muss das Bergrecht Anreize zur schonenden und nachhaltigen Nutzung von Rohstoffen setzen - beispielsweise über den Preis.

Dafür gilt es, alle Ausnahmen und Erleichterungen bei der Förderabgabe zu streichen und die im BBergG vorgesehene Förderabgabe von zehn Prozent des Marktwerts konsequent auf alle Rohstoffe zu erheben. Gleichzeitig fehlt es an Investitionsanreizen beispielsweise für konsequente Kreislaufführung von Stoffen und Produkten. Kreislaufwirtschaft schafft wirtschaftliche Innovation, nachhaltigen Wohlstand und Beschäftigung.

\section{AUTOR + KONTAKT}

Der Politikwissenschaftler Daniel Hiß leitet das Projekt „Umwelt- und Ressourcenschutz und Reform des Bundesberggesetzes" beim Deutschen Naturschutzring.

Daniel Hiß, Deutscher Naturschutzring. Tel.: +4930 6781775-72,

E-Mail: daniel.hiss@dnr.de, Internet: www.dnr.de/projekte/ reform-des-bundesberggesetzes/index.html

\section{Aus den Anden für die Welt}

Hier das "Bruttosozialglück", dort "Sumak kawsay": Nach Bhutan hat nun auch Ecuador seine eigene Formel für Glück gefunden. Sumak kawsay lautet in der indigenen Sprache Quechua die Entsprechung für das Gute Leben, auf Spanisch "Buen viviru. Dank Acosta haben nun zentrale Aspekte der Weltanschauung der Andenvölker Verfassungsstatus erlangt. In seinem Buch liefert er zahlreiche Denkanstöße für ein neues Verständnis von Gesellschaft, Natur und Wirtschaft.

\section{Alberto Acosta}

\section{Buen vivir}

Vom Recht auf ein gutes Leben 224 Seiten, Broschur, 16,95 Euro, ISBN 978-3-86581-705-1

Auch als E-Book erhältlich.

Beziehbar im Buchhandel oder versandkostenfrei bestellbar innerhalb

Deutschlands unter www.oekom.de

\section{II oekom}

Die guten Seiten der Zukunft

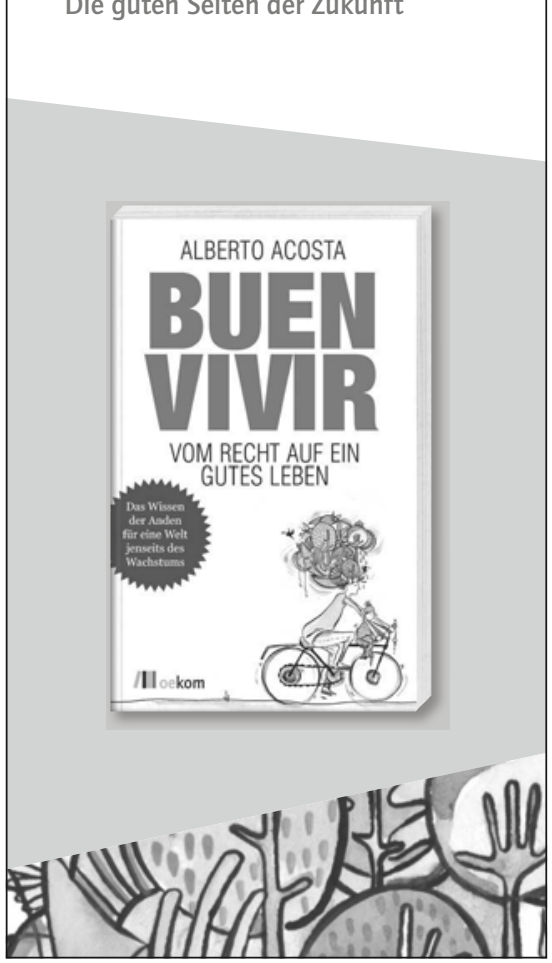

